\title{
Approximate Lattice Thermal Conductivity of MAX Phases at High Temperature
}

\author{
Chandra Dhakal, Sitaram Aryal, Ridwan Sakidja,and Wai-Yim Ching” \\ Department of Physics and Astronomy, University of Missouri - Kansas City, Kansas city, \\ Missouri, 64110, USA \\ \# Corresponding author: Chingw@ umkc.edu, Tel: 816-235-2503, Fax: 816-235-5221
}

\begin{abstract}
The temperature dependent lattice thermal conductivity $\left(\kappa_{p h}\right)$ of MAX phases, $\mathrm{M}_{n+1} \mathrm{AX}_{\mathrm{n}}$ are calculated using the Debye theory as outlined by Slack. At high temperature the formula derived by Slack is a reasonable approximation to estimate the lattice thermal conductivity. The calculation used the large data base of elastic coefficients of stable MAX phases established recently. It is found that MAX phases with " $\mathrm{A}$ " $=\mathrm{Al}$ have higher $\kappa_{p h}$ at $1300 \mathrm{~K}$, and the majority of MAX carbides have higher $\kappa_{p h}$ than MAX nitrides. We have also calculated the minimum thermal conductivities of these MAX phases using the empirical formula suggested by Clarke. It is shown that the minimal lattice thermal conductivities of MAX carbides and nitrides are closer to each other in the 211 phases than in higher $\mathrm{n}$ phases. The calculated $\kappa_{p h}$ for 8 MAX phases at $1300 \mathrm{~K}$ are in reasonable agreement with experimental data, especially in $\mathrm{Ti}_{2} \mathrm{AlC}, \mathrm{Nb}_{4} \mathrm{AlC}_{3}$, $\mathrm{Ta}_{4} \mathrm{AlC}_{3}, \mathrm{Nb}_{2} \mathrm{AlC}$ and $\mathrm{Nb}_{2} \mathrm{SnC}$ phases.
\end{abstract}

Key words: MAX phase, Lattice thermal conductivity, Minimal thermal Conductivity, temperature dependent thermal conductivity.

\section{Introduction}

MAX phases are a new class of ternary layered transition metal carbides and nitrides with a general formula $\mathrm{M}_{\mathrm{n}+1} \mathrm{AX}_{\mathrm{n}}$ (MAX), where $\mathrm{n}=1,2,3$ or higher discovered in 1960's [1, 2]. Here $\mathrm{M}$ is an early transitional metal, A is a group III, IV, V or VI element and X is either C or N. Depending on the value of layer index n, MAX phases are labeled as 211, 312 and 413 phases for $n=1,2,3$, respectively. Fig. 1 shows the sketches of the 211, 312, 413 PAX phases. In recent years, material scientists have been attracted to study MAX phases due to their unique combination of structural characteristics, wide range of properties, and many potential applications. MAX phases behave both like ceramics and metals with special desirable properties, such as machinable, thermal shock resistant, damage tolerant, fatigue, creep, oxidation resistant and are elastically stiff. They are also good thermal and electrical conductors [3-8]. There are more than 70 confirmed MAX phases with the majority of them being 211 carbides. 
The thermal conductivity of MAX phases is one of their most fundamental and important physical properties, especially for applications at elevated temperatures. Rigorous, first-principles calculation of thermal conductivity for complex crystals, such as MAX phases having dual properties of metals and ceramics, is extremely difficult. An alternative approach based on simpler procedure with justifiable approximations is therefore highly desirable. MAX phases are good thermal conductors. Their room temperature total thermal conductivity varies from 12 to $60 \mathrm{Wm}^{-1} \mathrm{~K}^{-1}$ [3]. The present work focuses on the calculation of lattice thermal conductivity of a large number of MAX phases by using Slack's equation based on Debye model [9] with particular emphasis on high temperature values, and in comparing with the minimal thermal conductivity using Clarke's formula [10]. Moreover, we identify the trends and differences in lattice thermal conductivity between MAX carbides and nitrides. We first discuss the rational for the approximations used in the calculation.

The ability to characterize heat transfer and its rate in a material depends upon the temperature gradient and thermal conductivity of the material. When heat current propagates through the specimen via charge carriers (electrons) and lattice vibrations (phonons), they diffuse and encounter random collisions along the way. In metals, the concentration of conduction electrons is high and almost all of heat conduction takes place via motion of electron. Hence, the electronic thermal conductivity $\left(\kappa_{e}\right)$ dominates over the lattice thermal conductivity $\left(\kappa_{p h}\right)$ by phonons. If impure atoms are present, especially in the form of solid solutions, [11] they act as scattering centers and reduce the total thermal conductivity $\kappa_{t h}=\kappa_{e}+\kappa_{p h}$. In insulators, the density of conduction electrons is negligible and conduction of heat energy is carried mostly by phonons and $\kappa_{p h}$ dominates. Thus, $\kappa_{p h}$ represents the major component of the overall thermal conductivity in ceramics. Phonons can also be scattered by lattice imperfections such as impurities, porosity and disordered structures, which reduce the lattice thermal conductivity.

Normally, the source of lattice thermal conductivity in a crystal is the sum of long range and short range acoustic phonons and to a certain extent, the optical phonons $[12,13]$. This is not always true for crystals with low thermal conductivity. At high temperatures, we are mainly focused on the phonon-phonon scattering, where anharmonic umklapp (U-) processes are instrumental in generating the resistance by reversing the direction of the wave vector when long range acoustic modes are considered [14]. Optical phonon modes do not directly take part in heat conduction but may interact with heat carrying acoustic phonons, which are more important in determining the magnitude of thermal conductivity [15]. The equation derived by Slack [9] is valid only when the critical frequency $\left(\omega_{c}\right)$ is comparable to the Debye frequency $\left(\omega_{\mathrm{D}}\right)$. The critical frequency $\left(\omega_{\mathrm{c}}\right)$ is the maximum vibrational frequency of a given mode in a crystal that can be obtained from the acoustic branches of the vibrational spectrum. Slack's equation was based on simple consideration of averaging the atoms of a "molecule" (or the atoms in the formula unit of the crystal) and their average atomic weight in simplifying the calculation for $\kappa_{p h}$. He realized that real materials are not perfect and may contain point defects, dislocations and other imperfections, which decrease their thermal conductivity. Slack's approach is to estimate the effect of defects analogous to the Rayleigh scattering of light [16]. He argued that the crystals containing isolated defects will have defect size smaller than long phonon wavelength and the phonon mean free path should be around one half of that [13]. The defect scatters a large number of phonons similar to the scattering of light by particles. One complication with this approach is that the average wavelength of phonons decreases with increase in temperature such that the phonon wavelength is no longer greater than the defect size which may not be below the Debye temperature. 
In this paper, we report a systematic calculation of lattice thermal conductivity $\left(\kappa_{p h}\right)$ and minimum thermal conductivity $\left(\kappa_{\min }\right)$ for a large number of 211,312 and 413 MAX phases by using Slack's equation and Clarke's formula respectively. To obtain the parameters used in these calculations (see next section), we take advantage of the recently established data base of fully relaxed crystal structures and the elastic coefficient $\left(\mathrm{C}_{\mathrm{ij}}\right)$, bulk mechanical properties, and equilibrium volume of all stable MAX phase compounds [17]. In the next section, the steps for the simplified methods are presented. In section 3, we present the calculated results with discussions. The calculated values for MAX carbides and nitrides are compared and contrasted for different layer index $n$ and for different "A" members. Finally, we compare our calculations with limited data reported from experimental measurements, which shows some reasonable agreement for some of them. The paper ends with some brief conclusions.

\section{Computational Steps:}

In this section, we describe the steps used in detertmining temperature dependent lattice phonon thermal conductivity of MAX phases in using Slack's equation. Similarly, Clarke formula is very useful to estimate the minimum thermal conductivity. We outline the steps employed in obtaining the input parameters used in the calculation. The density $\rho$ of MAX phase compound is calculated from fully relaxed unit cell volume [17]. We then use the bulk mechanical parameters from our data base (bulk modulus (B), shear modulus (G)) to obtain the longitudinal, trasverse and average sound velocity $\mathrm{v}_{\mathrm{l}}, \mathrm{v}_{\mathrm{t}}$, and $\mathrm{v}_{\mathrm{m}}$ repectively $[18,19]$.

$$
\begin{aligned}
& \mathrm{v}_{\mathrm{l}}=\left(\frac{3 \mathrm{~B}+4 \mathrm{G}}{3 \rho}\right)^{1 / 2} \text { and } \mathrm{v}_{\mathrm{t}}=\left(\frac{\mathrm{G}}{\rho}\right)^{1 / 2} \\
& \mathrm{v}_{\mathrm{m}}=\left[\frac{1}{3}\left(\frac{2}{\mathrm{v}_{\mathrm{t}}^{3}}+\frac{1}{\mathrm{v}_{1}^{3}}\right)\right]^{-1 / 3}
\end{aligned}
$$

The average phonon velocity $v_{m}$ is used to estimate the Debye temperature $\theta_{D}$. Debye temperature is one of the most fundamental parameters in characterizing the vibrational properties of a material which is also closely related to other physical properties such as elastic constants, specific heat and melting temperature. Generally speaking, higher Debye temperature implies larger value of thermal conductivity but this is not always true. The vibrational excitations modes at low temperature arise exclusively from acoustic modes. Hence, $\theta_{\mathrm{D}}$ calculated from elastic parameters should be the same as those obtained from the specific heat measurements at low temperature. One of the accepted practice is to obtain $\theta_{\mathrm{D}}$ from average phonon velocity $\mathrm{v}_{\mathrm{m}}$ by using the following expression [20].

$$
\theta_{\mathrm{D}}=\frac{\mathrm{h}}{\mathrm{k}_{\mathrm{B}}}\left\lfloor\frac{3 \mathrm{n}}{4 \pi}\left(\frac{\rho \mathrm{N}_{\mathrm{A}}}{\mathrm{M}}\right)\right\rfloor \mathrm{v}_{\mathrm{m}}
$$

where $h, k_{B}, N_{A}, n$ and $M$ are the Plank's constant, Boltzmann constant, Avogadro's number,number of atoms per molecule and molecular mass, respectively. 
Finally, to calculate temperature dependent lattice thermal conductivity $\kappa_{p h}$, the following empirical equation derived by Slack [21] is used,

$$
\kappa_{p h}=A \frac{\bar{M} \theta_{D}^{3} \delta}{\gamma^{2} n^{2 / 3} T}
$$

where $\bar{M}$ is the average atomic weight (in unit of $\mathrm{kg} / \mathrm{mol}$ ), $\delta^{3}$ is the average volume (in unit of $\mathrm{m}^{3}$ ) of the mass equivalent one atom in the primitive unit cell, $\mathrm{T}$ is the absolute temperature, $\mathrm{n}$ is number of atoms per unit cell, $\gamma$ is the Grüneisen parameter obtained from Poison's ratio (v) and A is a coefficient depending on $\gamma$ (in unit of $\mathrm{W} \mathrm{mol} / \mathrm{kg} / \mathrm{m}^{2} / \mathrm{K}^{3}$ ). The Grüneisen parameter can partially address the issue of anharmonicity of phonon-phonon interaction [22].

$$
\gamma=\frac{9\left(v_{1}^{2}-\frac{4}{3} v_{t}^{2}\right)}{2\left(v_{1}^{2}-2 v_{t}^{2}\right)}=\frac{3}{2}\left(\frac{1+v}{2-3 v}\right)
$$

The Grüneisen parameter is defined by $\gamma\left(\omega_{\mathrm{n}}\right)=\mathrm{d}\left(\ln \omega_{\mathrm{n}}\right) / \mathrm{d}(\ln \varphi)$ where $\omega_{n}$ and $\varphi$ are frequency and packing fraction of the crystal. It controls the number of physical process including thermal conductivity and temperature dependent elastic properties. The packing fraction of crystal gives us the basic information about the arrangements of atoms in unit cell and its stability. The vibrating atoms in a tightly packed crystals act as rattlers which has significant effect on lattice thermal conductivity [23, 24]. The coefficient $\mathrm{A}(\gamma)$ in Eg. (4) was determined by Julian [25] to be:

$$
\mathrm{A}(\gamma)=\frac{5.720 \times 10^{7} \times 0.849}{2 \times\left(1-\frac{0.514}{\gamma}+\frac{0.228}{\gamma^{2}}\right)}
$$

More recently, Clark proposed that a material at high temperature possesses a minimum thermal conductivity. Materials with a very low thermal conductivity are good candidate for thermal barrier coating (TBC) [26]. He used a slightly different argument from that of Slack and with additional approximations, starting from the thermal conductivity expression from the kinetic theory of gases [24].

$$
\kappa=\frac{\mathrm{C}_{\mathrm{v}} \mathrm{v}_{\mathrm{m}} \Lambda}{3}
$$

where $\mathrm{C}_{\mathrm{v}}$ is the specific heat at constant volume and $\mathrm{v}_{\mathrm{m}}$ is the mean phonon velocity and $\Lambda$ is the mean free path of phonon [27]. At high temperature T, the phonon mean free path $\Lambda \propto 1 / \mathrm{T}$ is limited to the order of lattice constant, or $\kappa \propto 1 / \mathrm{T}$ [28]. The mean free path would be infinite in the harmonic approximation of lattice dynamics of phonons.

The intrinsic minimum thermal conductivity is the lowest value of thermal conductivity for a perfect crystal at high temperature when phonons are completely uncoupled and heat energy is transferred 
between neighboring atoms [29]. In this limit, the phonons mean free path can be approximated to be the average interatomic distance. Accordingly, one can replace different atoms in a molecule with an equivalent atom having the mean atomic mass of $\mathrm{M} / \mathrm{n}(\mathrm{n}=$ number atoms in the unit cell). The same approximation has been used in calculation of thermal conductivity of molecules nearly 50 year ago [30]. A single "equivalent atom" in the cell does not have any optical modes and can be used obtain a formula $\kappa_{\min }$ at high temperature given by [10]

$$
\kappa_{\text {min }}=\mathrm{k}_{\mathrm{B}} \mathrm{v}_{\mathrm{m}} \Lambda_{\text {min }}=\mathrm{k}_{\mathrm{B}} \mathrm{v}_{\mathrm{m}}\left(\frac{\mathrm{M}}{\mathrm{n} \rho \mathrm{N}_{\mathrm{A}}}\right)^{-2 / 3}
$$

Where $\Lambda_{\min }, \mathrm{N}_{\mathrm{A}}$ and $\rho$ are the phonon mean free path, Avogadro's constant and density of crystal respectively. We used this formula to estimate $\kappa_{\min }$ for the MAX phases.

\section{Results and Discussion}

In this section, we present the results of lattice thermal conductivity and the minimum thermal conductivity of the 211, 312 and 413 MAX phases using the methods described above. In Figure 2, we show the calculated values of $\kappa_{p h}$ at $1300 \mathrm{~K}$ on the 551 screened MAX phases [17], using Slack's approach. The same data are plotted in two different ways in order to trace the trends with variations in the atomic numbers of " $M$ " and " $A$ " elements. The left column in Figure 2 is for "M"-based plots and the right column is for the " $\mathrm{A}$ "-based plots. The $\mathrm{X}$-axis lists the 9 different " $\mathrm{M}$ " elements and 11 " $\mathrm{A}$ " elements for the two columns respectively. The data for carbides (solid circle) and nitrides (open circle) are plotted in the same figure. In the following subsection, we discuss these results for $\kappa_{p h}$ at $1300 \mathrm{~K}$ for the 211, 312 and 413 MAX phases.

\subsection{Lattice thermal conductivity at 1300K for MAX phases using Slack's formula}

Slack's equation is very effective in calculation of phonon thermal conductivity, especially for nonmetals. We used this formula, Eq. (4) to obtain $\kappa_{p h}$ of all stable 211, 312 and 413 MAX phases at 1300K. The results are shown in Figure 2. This is a very busy figure because of the amount of data involved. To grasp the variations and overall trends in these $\kappa_{p h}$ values more easily, we employ the following strategy: (1) the data for carbides (solid circles) and nitrides (open circles) are plotted on the same figure. (2) The horizontal $\mathrm{x}$-axis is in the increasing order of atomic number $\mathrm{Z}$. The left column is for " $\mathrm{M}$ " ( $\mathrm{Sc}, \mathrm{Ti}, \mathrm{V}, \mathrm{Cr}$, $\mathrm{Zr}, \mathrm{Nb}, \mathrm{Mo}, \mathrm{Hf}$ and Ta) and the right column is for "A" group elements (Al, Si, P, S, Ga, Ge, As, In, Sn, $\mathrm{Tl}$ and $\mathrm{Pb}$ ). They are separated by vertical blocks of differently shaded colors. Each colored area encloses 22 MAX phases with different " $A$ " in the left column and 16 MAX phases with different ' $M$ ' in the right column. (3) The order of both ' $A$ ' and ' $M$ ' in each block is again in the increasing order of their atomic numbers. The left and right panels contain the same amount of data points but plotted in different ways to facilitate the observation in the trends for different " $M$ " and different " $A$ ". (4) The vertical scale (0 to 20 $\mathrm{Wm}^{-1} \mathrm{~K}^{-1}$ ) for $\kappa_{p h}$ is kept the same for all 6 panels for easy comparison. Despite the overwhelming amount of the data, the following trends and observations can be discerned:

(1) The MAX phases with 5 highest $\kappa_{p h}$ at $1300 \mathrm{~K}$ are all nitrides: The values are 19.14, 17.33, 16.96, 15.83 and $14.71 \mathrm{Wm}^{-1} \mathrm{~K}^{-1}$ for $\mathrm{Ti}_{2} \mathrm{AlN}, \mathrm{V}_{2} \mathrm{AlN}, \mathrm{Ti}_{2} \mathrm{PN}, \mathrm{Sc}_{2} \mathrm{SN}$ and $\mathrm{Ti}_{2} \mathrm{SN}$ respectively. The lowest $5 \kappa_{p h}$ values at $1300 \mathrm{~K}$ are $0.141,0.097,0.087,0.086$ and 0.048 in the same unit corresponding to $\mathrm{Ta}_{4} \mathrm{SnN}_{3}$, 
$\mathrm{Cr}_{2} \mathrm{PbC}, \mathrm{Ta}_{3} \mathrm{SnN}_{2}, \mathrm{~V}_{3} \mathrm{PbN}_{2}$ and $\mathrm{Ta}_{2} \mathrm{PbN}$. Only 1 carbide is among the 5 MAX phases with lowest $\kappa_{p h}$. Thus MAX nitrides have more scattered range of $\kappa_{p h}$ at $1300 \mathrm{~K}$.

(2) In the panels for different ' $M$ ' on the left column, the Sc-based MAX phases (first panel to the left) in 211 phases are more widely dispersed than the data from 312 and 413 phases. In the same Sc panel, the values for carbides are much smaller than the nitrides. For other M panels, nitrides have lower $\kappa_{p h}$ than carbides except in the 211 MAX phases where they are mixed. There is also the obvious trend of reduced $\kappa_{p h}$ as $\mathrm{Z}$ increases within each panel. This observation is much more pronounced on the left column (variation of " $A$ " in each panel for " $M$ ") than on the right panel (variation of "M" in each panel for "A"). It means that variation in " $\mathrm{A}$ " is the major factor in the trends for $\kappa_{p h}$ and they show more distinctive separations between carbides and nitride with later having higher $\kappa_{p h}$ except for "M" $=$ Sc. The trend of reduced $\kappa_{p h}$ as atomic number $\mathrm{Z}$ of " $\mathrm{M}$ " increases is much reduced as can be seen on the right column plots. This is contrary to the notion that " $\mathrm{M}$ " should be more pronounced than " $\mathrm{A}$ " since they are more "M" elements than " $A$ " elements in MAX phases.

(3) In the panels for different " $A$ " on the right column, the data for carbides and nitrides for all MAX phases are rather scattered. Within each panel for fixed "A", the trends of decreasing $\kappa_{p h}$ with increasing Z value of "M" is not that obvious which is in striking contrast with that data on the left column for the variation of "A" for fixed "M".

(4) From observation of the data in Figure 2, we found that $\kappa_{p h}$ are more widely distributed and have larger values in 211 MAX phases. They are much smaller and more narrowly distributed in the 312 and 413 phases Thus it is safe to say that $\kappa_{p h}$ generally scales inversely with the layer index $\mathrm{n}$.

The above observations provide an overall view of the lattice thermal conductivity of a large number of MAX phases. We are not aware of any such information in the published literature.

\subsection{Minimum thermal conductivity of MAX phases using Clarke's formula}

The Clark's formula is very useful to obtain the temperature independent minimum thermal conductivity, $\kappa_{\min }$. We have calculated $\kappa_{\min }$ for the same 551 MAX phases in the data base using Eq. (8). The results are shown in the left column of Figure 3 with values of $\kappa_{\min }$ for MAX carbides vs. MAX nitrides plotted. (114 of the 551 phases were excluded because we must have both data for carbides and nitrides of the same phase be in the data base). It shows that the majority of carbides have larger $\kappa_{\min }$ than nitrides in all MAX phases. In other words, there are more data points below the diagonal line. For the 211 phase, the data points are closer to the diagonal line indicating less difference in $\kappa_{\min }$ between carbides and nitrides. This is in opposite direction from the data shown in Fig. 2 for 211 phases at 1300K calculated using Slack formula. As $n$ increases, $\kappa_{\min }$ move away from the diagonal line signaling a larger difference in $\kappa_{\min }$ between carbides and nitrides. We note that the following $211 \mathrm{MAX}$ carbides $\mathrm{Ti}_{2} \mathrm{GaC}, \mathrm{Sc}_{2} \mathrm{PC}, \mathrm{Nb}_{2} \mathrm{AlC}$, $\mathrm{Ta}_{2} \mathrm{AlC}, \mathrm{Cr}_{2} \mathrm{TlC}, \mathrm{Hf}_{2} \mathrm{InC}$ have almost the same $\kappa_{\min }$ as their corresponding nitrides. The largest differences in which $\kappa_{\min }$ for carbides are larger than nitrides in the 211 phases are Cr-based carbides $\left(\mathrm{Cr}_{2} \mathrm{AlC}\right.$, $\mathrm{Cr}_{2} \mathrm{SiC}, \mathrm{Cr}_{2} \mathrm{GaC}, \mathrm{Cr}_{2} \mathrm{GeC}, \mathrm{Cr}_{2} \mathrm{InC}$ ) whereas in $\mathrm{Ti}_{2} \mathrm{PN}, \mathrm{Ti}_{2} \mathrm{AsN}, \mathrm{Sc}_{2} \mathrm{GaN}, \mathrm{Cr}_{2} \mathrm{PbN}$ and $\mathrm{Ti}_{2} \mathrm{AsN}, \kappa_{\min }$ of nitrides are larger than their corresponding carbides. In $312 \mathrm{MAX}$ phases, the $\kappa_{\min }$ for some carbide $\left(\mathrm{Ti}_{3} \mathrm{SC}_{2}, \mathrm{Ti}_{3} \mathrm{PC}_{2}, \mathrm{Hf}_{3} \mathrm{InC}_{2}, \mathrm{Cr}_{3} \mathrm{TlC}_{2}, \mathrm{Zr}_{3} \mathrm{SC}_{2}\right)$ are nearly the same as the nitrides. The largest differences in which the carbides are larger than the nitrides are $\mathrm{Ta}_{3} \mathrm{SnC}_{2}, \mathrm{Ta}_{3} \mathrm{GeC}_{2}, \mathrm{Cr}_{3} \mathrm{GaC}_{2}, \mathrm{Cr}_{3} \mathrm{SiC}_{2}, \mathrm{Cr}_{3} \mathrm{AlC}_{2}$; and for 

phases, the carbides $\mathrm{Zr}_{4} \mathrm{GaC}_{3}, \mathrm{Zr}_{4} \mathrm{SnC}_{3}, \mathrm{Zr}_{4} \mathrm{AlC}_{3}, \mathrm{Hf}_{4} \mathrm{GaC}_{3}, \mathrm{Ti}_{4} \mathrm{GaC}_{3}$ are almost equal to their corresponding nitrides. The MAX carbides $\mathrm{Ta}_{4} \mathrm{SnC}_{3}, \mathrm{Cr}_{4} \mathrm{AlC}_{3}, \mathrm{~V}_{4} \mathrm{SC}_{3}, \mathrm{~V}_{4} \mathrm{PC}_{3}, \mathrm{~V}_{4} \mathrm{AlC}_{3}$ etc. have higher $\kappa_{\min }$ than corresponding nitrides and the nitrides $\mathrm{Sc}_{4} \mathrm{TlN}_{3}, \mathrm{Sc}_{4} \mathrm{InN}_{3}, \mathrm{Sc}_{4} \mathrm{GaN}_{3}, \mathrm{Sc}_{4} \mathrm{SiN}_{3}, \mathrm{Sc}_{4} \mathrm{AlN}_{3}$ etc. have higher $\kappa_{\min }$ than their corresponding carbides. This plethora of differences in carbides and nitrides in $\kappa_{\min }$ is truly astonishing. This can only be attributed to the diversity in composition, structure, electronic bonding in MAX phases as elaborated in reference [17].

We have pointed out earlier that the trend for $\kappa_{\min }$ in 211 phases is opposite to the data at $1300 \mathrm{~K}$ calculated using Slack formula. To gain some insight, we calculated the data using Slack's formula at an even higher temperature of $2000 \mathrm{~K}$. The results for carbides vs nitrides are plotted on the right column of Figure 3. It is fairly apparent that the trends are now similar as $\kappa_{\min }$ calculated using Clarke's formula on the left column. In order words, both $\kappa_{p h}$ data at $2000 \mathrm{~K}$ and $\kappa_{\min }$ data are found to be somewhat similar to each other. This illustrates that in MAX phases, the minimum lattice thermal conductivity should be at relative high temperature close to $2000 \mathrm{~K}$. It also demonstrates the results from Clarke's formula to be close to that predicted by Slack's equation at high temperature despite their different derivations and approximations.. We still could not explain such a wide variations in $\kappa_{\min }$ in MAX phases but the data could provide an overall picture of their minimal lattice thermal conductivity with potential implications on possible applications as TBC materials at high temperature [20]. The main conclusion is that generally speaking, MAX carbides have their minimal lattice thermal conductivity larger than the nitrides.

\subsection{Comparison with experimental data}

We have searched for available experimental data on lattice thermal conductivities of MAX phases at different temperatures. The data were quite scattered. We have located experimental data on eight MAX phases at $1300 \mathrm{~K}$ and $300 \mathrm{~K}$ from the recent book by Barsoum [3]. In Figure 3, we plotted the calculated $\kappa_{p h}$ vs. experimental $\kappa_{p h}$ at $1300 \mathrm{~K}$ for these eight crystals. They are all MAX carbides: $\mathrm{Ti}_{2} \mathrm{AlC}, \mathrm{Nb}_{4} \mathrm{AlC}_{3}$, $\mathrm{Ta}_{4} \mathrm{AlC}_{3}, \mathrm{Nb}_{2} \mathrm{AlC} \mathrm{Nb} \mathrm{SnC}_{2} \mathrm{Ta}_{2} \mathrm{AlC}, \mathrm{Cr}_{2} \mathrm{AlC}$ and $\mathrm{Ti}_{3} \mathrm{SiC}_{2}$. We note that six of these phases have "A" = Al. It can be seen that $\mathrm{Nb}_{4} \mathrm{AlC}_{3}$ lies exactly on the diagonal line in perfect agreement which is of course fortuitous. The other three phases $\left(\mathrm{Ti}_{2} \mathrm{AlC}, \mathrm{Ta}_{4} \mathrm{AlC}_{3}\right.$, and $\left.\mathrm{Nb}_{2} \mathrm{SnC}\right)$ are close to the diagonal line with experimental data slightly larger than the calculated values. On the other hand, the remaining four MAX phases $\left(\mathrm{Ti}_{3} \mathrm{SiC}_{2}, \mathrm{Cr}_{2} \mathrm{AlC}, \mathrm{Nb}_{2} \mathrm{AlC}\right.$, and $\left.\mathrm{Ta}_{2} \mathrm{AlC}\right)$ have experimental values about a factor 2 less than the calculated values. In fact, $\mathrm{Ta}_{4} \mathrm{AlC}_{3}$ and $\mathrm{Ti}_{2} \mathrm{AlC}$ are only $20 \%$ and $25 \%$ lower than the experimental values respectively, whereas $\mathrm{Cr}_{2} \mathrm{AlC}$ and $\mathrm{Nb}_{2} \mathrm{AlC}$ are a factor of 3 and 2 higher than the reported experimental value.

The $\kappa_{p h}$ of MAX phases containing $\mathrm{Al}$ atoms has some important implications because $\mathrm{Al}$ is the lightest atom on the " $\mathrm{A}$ " list. It is a more effective rattler, therefore scatters less phonons resulting in a higher lattice thermal conductivity. But in some 'Al' containing MAX phases such as in $\mathrm{Ta}_{2} \mathrm{AlC}$ and $\mathrm{Cr}_{2} \mathrm{AlC}$, the experimental $\kappa_{p h}$ are very small compared to calculated values. A possible explanation of this disparity is the presence of point defects or other disordered structures in the measured samples of these MAX phases [3] and the complication associated with the large electric component of in the total thermal conductivity. The higher $k_{p h}$ for $\mathrm{Ti}_{2} \mathrm{AlC}$ could be attributed to the quality of the sample in comparison with say $\mathrm{Nb}_{2} \mathrm{AlC}$ [31]. Also, experimental $\kappa_{p h}$ of $\mathrm{Ti}_{3} \mathrm{SiC}_{2}$ is very small as shown in Figure 4. According to Barsoum [3], its total thermal conductivity is actually quite high but the contribution from lattice vibration, or $\kappa_{p h}$ tends to be very low because of the much higher contribution from the electronic part of the thermal conductivity due to high quality of the sample [32]. Figure 4 also shows that the calculated $\kappa_{p h}$ of $\mathrm{Nb}_{2} \mathrm{SnC}$ is the smallest among the eight MAX phases, smaller than the experimental value. Since 
$\mathrm{Sn}$ is much heavier than $\mathrm{Al}$, it is expected that the $\kappa_{p h}$ for $\mathrm{Nb}_{2} \mathrm{SnC}$ should be smaller than Al-based MAX phases consistent with the trends in the calculated values discussed earlier and displayed in Figure 2.

It should be point out that accurate calculation for lattice thermal conductivity is extremely challenging and requires the knowledge of phonon spectrum and phonon density of states (DOS) for each of the MAX phases which are well beyond the scope of this paper. In Figure 5, we show the phonon spectra and phonon DOS for $5 \mathrm{MAX}$ phases $\left(\mathrm{Ti}_{2} \mathrm{AlC}, \mathrm{Ti}_{3} \mathrm{AlC}_{2}, \mathrm{Ti}_{2} \mathrm{AlN}, \mathrm{Cr}_{2} \mathrm{AlC}, \mathrm{Ti}_{3} \mathrm{SiC}_{2}\right.$, unpublished results) as illustration. Four of them have "A" = Al and the other one with "A" = Si. Two (three) of them are 312 (211) phases. The only MAX nitride $\mathrm{Ti}_{2} \mathrm{AlN}$ can be used to compare with MAX carbide $\mathrm{Ti}_{2} \mathrm{AlC}$. It can be seen that these phonon spectra can be roughly divided into two parts, the upper one from $\mathrm{X}$ atoms $(\mathrm{C}$ or $\mathrm{N}$ ) and lower one from " $\mathrm{M}$ " and "A" elements which overlap and with the heavier " $\mathrm{M}$ " phonon bands at lower frequency including all the acoustic phonon branches. These two segments of the phonon bands are separated by a large gap in the frequency range varying from $150-250 \mathrm{~cm}^{-1}$. The phonon spectra can also depend on the layer index n." Unfortunately, we are not able to provide the phonon spectra for all the MAX phases discussed in this paper.

Given the fact that experimental measurement and extraction of lattice thermal conductivity in MAX phases is notoriously difficult and there are large variations depending on the nature the samples used, and the calculations presented here are based on simple models, the comparison between the two shown in Figure 3 can be considered to be very encouraging.

In Table 1, we list the results of the minimal thermal conductivity $\kappa_{\min }$ of the same 8 MAX phases and the parameters used in the calculation $\left(\Theta_{\mathrm{D}}, \gamma, \operatorname{Vol}\left(\AA^{3}\right), \mathrm{M}(\mathrm{amu})\right)$ together with values of calculated $\kappa_{p h}$ and experimental $\kappa_{p h}$ at $1300 \mathrm{~K}$. It can be seen that the calculated $\kappa_{\min }$ are much lower than the calculated and measured $\kappa_{p h}$ at $1300 \mathrm{~K}$ as already discussed in section 3.2.

\subsection{Temperature dependence phonon thermal conductivity $\left(\kappa_{P h}\right)$ of selected MAX phases}

Although our focus on this paper is the lattice thermal conductivity for MAX phases at high temperature, the Slack formula used was actually derived for any temperature. It is therefore instructive to at least calculate the temperature dependence of the lattice thermal conductivity for some of the MAX phases. To this end, we choose the eight MAX phases discussed above to show the calculated temperature dependence of $\kappa_{p h}$. The results are presented in Figure 6. As can be seen, at low temperature, the lattice $\kappa_{p h}$ can be as high as above $80 \mathrm{Wm}^{-1} \mathrm{~K}^{-1}$ in $\mathrm{Ti}_{2} \mathrm{AlC}$. They steadily decrease as temperature increases. Also shown in Figure 4 are the reported data at 300K. They are naturally much less than the calculated values since our assumptions and justifications are geared towards the calculation of lattice thermal conductivity at high temperatures. However, in the case of $\mathrm{Ta}_{4} \mathrm{AlC}_{3}$, the calculated $\kappa_{p h}$ at $300 \mathrm{~K}$ is only slightly higher than the measure value of about $18 \mathrm{Wm}^{-1} \mathrm{~K}^{-1}$ and the data at $1300 \mathrm{~K}$ and is also in close agreement with the measure data. Thus $\mathrm{Ta}_{4} \mathrm{AlC}_{3}$ is obviously an outlier in the trend observed. Additional investigation on the thermal conductivity for $\mathrm{Ta}_{4} \mathrm{AlC}_{3}$, is clearly warranted. In Figure 6, we also plot the minimal thermal conductivities $\kappa_{\min }$ as dashed lines for these MAX phases as discussed in 3.2. They are very close or slightly below the calculated $\kappa_{p h}$ at $2000 \mathrm{~K}$.

\section{Conclusions}

We have calculated the lattice thermal conductivity $\left(k_{p h}\right)$ of 551 MAX phase compounds using the simple equation derived by Slack and the published elastic coefficients on MAX phases. The data are analyzed 
on the basis of "M" and " $\mathrm{A}$ " trends with increasing order of their atomic number Z, where $k_{p h}$ decreases with $\mathrm{Z}$ in general. This trend is more pronounced in variation with $\mathrm{Z}$ of " $\mathrm{A}$ " than with $\mathrm{Z}$ of " $\mathrm{M}$ ". We note that $k_{p h}$ of MAX phases decrease with increase in layer index ' $\mathrm{n}$ '. The calculated $k_{p h}$ for nitrides are found to be more scattered. We used Clarke's formula to obtain minimum thermal conductivity of these MAX phases and find MAX carbides have higher values of $\kappa_{\text {min }}$. Despite many approximations used in this study, the calculated lattice thermal conductivity of $\mathrm{Nb}_{4} \mathrm{AlC}_{3}$ is found to be in good agreement with experimental data at $1300 \mathrm{~K}$ and in reasonable agreement for $\mathrm{Ti}_{2} \mathrm{AlC}, \mathrm{Nb}_{4} \mathrm{AlC}_{3}, \mathrm{Ta}_{4} \mathrm{AlC}_{3}, \mathrm{Nb}_{2} \mathrm{AlC}$ and $\mathrm{Nb}_{2} \mathrm{SnC}$. In particular, the calculated $k_{p h}$ of $\mathrm{Ta}_{4} \mathrm{AlC}_{3}$ at $300 \mathrm{~K}$ is only slightly greater than the experimental values. Our study is the first time estimates revealing the general trends in the lattice thermal conductivities at high temperatures for a large collection of MAX phase compounds.

\section{Acknowledgements}

* This work was supported by National Energy Technology Laboratory (NETL) of the U.S. Department of Energy (DOE) under Grant No. DE-FE0005865. This research used the resources of the National Energy Research Scientific Computing Center (NERSC) supported by the Office of Basic Science of DOE under Contract No. DE-AC03-76SF00098.

Table 1. Calculated values of $\kappa_{\min }, \Theta_{\mathrm{D}}, \gamma, \kappa_{p h}$, volume, mass, number of atoms of unit cell of 8 MAX phases and experimental value of $\kappa_{p h}$.

\begin{tabular}{|c|l|l|l|l|l|c|c|l|}
\hline Compounds & $\mathrm{n}$ & $\Theta_{\mathrm{D}}(\mathrm{K})$ & $\Gamma$ & $\mathrm{Vol}\left(\AA^{3}\right)$ & $\mathrm{M}(\mathrm{amu})$ & $\begin{array}{c}\kappa_{p h}(\mathrm{cal}) \\
\left(\mathrm{Wm}^{-1} \mathrm{~K}^{-1}\right)\end{array}$ & $\begin{array}{l}\kappa_{p h}(\mathrm{exp}) \\
\left(\mathrm{Wm}^{-1} \mathrm{~K}^{-1}\right)\end{array}$ & $\begin{array}{l}\kappa_{\min } \\
\left(\mathrm{Wm}^{-1} \mathrm{~K}^{-1}\right)\end{array}$ \\
\hline $\mathrm{Ti}_{2} \mathrm{AlC}$ & 8 & 716 & 1.230 & 112.02 & 134.73 & 13 & 16 & 1.378 \\
\hline $\mathrm{Ta}_{2} \mathrm{AlC}$ & 8 & 449 & 1.448 & 115.58 & 400.89 & 7 & 0 & 0.855 \\
\hline $\mathrm{Nb}_{2} \mathrm{AlC}$ & 8 & 576 & 1.395 & 118.22 & 224.80 & 8 & $>4$ & 1.089 \\
\hline $\mathrm{Nb}_{2} \mathrm{SnC}$ & 8 & 412 & 1.689 & 129.35 & 316.53 & 3 & 5 & 0.755 \\
\hline $\mathrm{Cr}_{2} \mathrm{AlC}$ & 8 & 744 & 1.312 & 88.94 & 142.98 & 12 & 4 & 1.545 \\
\hline $\mathrm{Ti}_{3} \mathrm{SiC}_{2}$ & 12 & 795 & 1.324 & 145.25 & 195.70 & 10 & 1 & 1.607 \\
\hline $\mathrm{Ta}_{4} \mathrm{AlC}_{3}$ & 16 & 487 & 1.462 & 206.60 & 786.80 & 5 & 6 & 0.963 \\
\hline $\mathrm{Nb}_{4} \mathrm{AlC}_{3}$ & 16 & 644 & 1.374 & 210.71 & 434.64 & 7 & 7 & 1.265 \\
\hline
\end{tabular}




\section{Figure Captions}

Figure 1. Sketch of crystal structures of four $M A X$ phases $M_{2} A X, M_{3} A X_{2}, M_{4} A X_{3}$, (i.e. with $n$ $=1,2,3$.)

Figure 2. Scatter plots of calculated phonon thermal conductivity $\left(\kappa_{p h}\right)$ at $1300 \mathrm{~K}$ of MAX phases (a) 211 in "M" trend; (b) 211 in "A" trend; (c) 312 in "M" trend; (d) 312 in " $A$ " trend; (e) 413 in "M" trend (f) 413 in "A" trend. The trend for "M" elements ( $\mathrm{Sc}, \mathrm{Ti}, \mathrm{V}, \mathrm{Cr}, \mathrm{Zr}, \mathrm{Nb}, \mathrm{Mo}, \mathrm{Hf}$ and $\mathrm{Ta}$ ) and "A" elements (Al, Si, P, S, Ga, Ge, As, In, Sn, Tl and $\mathrm{Pb}$ ) are along the $\mathrm{x}$ - axis in left and right side of figures, respectively. Each panel of different colors contain 22 and 16 MAX phase compounds for the left and right side of figures respectively.

Figure 3. Left column: calculated minimum thermal conductivity $\kappa_{\min }$ of MAX carbides vs. MAX nitrides: (a) 211, (b) 312, and (c) 413. Rigt column: calculated $\kappa_{p h}$ at 2000K of MAX carbides vs. MAX nitrides: (d) 211, (e) 312, and (f) 413. Both x- and y-axes of each figure have the same scale. Data points on the diagonal lines indcate the $\kappa_{\min }$ or $\kappa_{p h}$ for MAX carbides and nitrides are equal.

Figure 4. Calculated phonon thermal conductivity of eight MAX phases $\left(\mathrm{Nb}_{2} \mathrm{AlC}, \mathrm{Nb}_{2} \mathrm{SnC}\right.$, $\mathrm{Ta}_{2} \mathrm{AlC} \mathrm{Ti}_{2} \mathrm{AlC}, \mathrm{Cr}_{2} \mathrm{AlC}, \mathrm{Nb}_{4} \mathrm{AlC}_{3}, \mathrm{Ta}_{4} \mathrm{AlC}_{3}$ and $\mathrm{Ti}_{3} \mathrm{SiC}_{2}$ ) at $1300 \mathrm{~K}$ vs. experimental data at same temperature.

Figure 5. Calcated phonon dispersion relation and density of states for five MAX phases: $\mathrm{Ti}_{2} \mathrm{AlC}$, $\mathrm{Ti}_{3} \mathrm{AlC}_{2}, \mathrm{Ti}_{2} \mathrm{AlN}, \mathrm{Cr}_{2} \mathrm{AlC}, \mathrm{Ti}_{3} \mathrm{SiC}_{2}$.

Figure 6. Calculated temperature dependent phonon thermal conductivity for eight MAX phases in Figure 3. (a) $\mathrm{Nb}_{2} \mathrm{AlC}$, (b) $\mathrm{Ta}_{2} \mathrm{AlC}$, (c) $\mathrm{Nb}_{2} \mathrm{SnC}$, (d) $\mathrm{Ti}_{2} \mathrm{AlC}$, (e) $\mathrm{Cr}_{2} \mathrm{AlC}$, (f) $\mathrm{Ti}_{3} \mathrm{SiC}_{2}$, (g) $\mathrm{Ta}_{4} \mathrm{AlC}_{3}$ and $(\mathrm{h}) \mathrm{Nb}_{4} \mathrm{AlC}_{3}$. Red dots are calculated data and blue dots are experimental value from ref. [3]. The horizontal dash line shows the calculated minimum phonon conductivity $\kappa_{\min }$. 


\section{References}

1. Barsoum, M. W. and El-Raghy T., The MAX phases: unique new carbide and nitride materials. American Scientist. 2001, 89(4), 336-45.

2. Nowotny, V. H., Strukturchemie einiger Verbindungen der Übergangsmetalle mit den elementen C, Si, Ge, Sn. Progress in Solid State Chemistry. 1971, 5, 27-70.

3. Barsoum, M. W., MAX Phases: Properties of Machinable Ternary Carbides and Nitrides: John Wiley \& Sons, 2013.

4. Wang, J. and Zhou, Y., Recent progress in theoretical prediction, preparation, and characterization of layered ternary transition-metal carbides. Annual Review of Materials Research. 2009, 39, 415-43.

5. Skarvelis, P. and Papadimitriou, G., Plasma transferred arc composite coatings with selflubricating properties, based on Fe and Ti sulfides: Microstructure and tribological behavior. Surface and Coatings Technology. 2009, 203(10), 1384-94.

6. Sun, Z., Hashimoto, H., Tian, W. and Zou, Y., Synthesis of the MAX phases by pulse discharge sintering. International Journal of Applied Ceramic Technology. 2010, 7(6), 704-18.

7. Gilbert, C., Bloyer, D., Barsoum, M., El-Raghy, T., Tomsia, A. and Ritchie, R., Fatigue-crack growth and fracture properties of coarse and fine-grained $\mathrm{Ti}<\mathrm{sub}>3</ \mathrm{sub}>\mathrm{SiC}<\mathrm{sub}>2</$ sub $>$. Scripta materialia. 2000, 42(8), 761-7.

8. Racault, C., Langlais, F. and Naslain, R., Solid-state synthesis and characterization of the ternary phase Ti3SiC2. Journal of materials Science. 1994, 29(13), 3384-92.

9. Slack, G. A., The thermal conductivity of nonmetallic crystals. Solid state physics. 1979, 34, 1-71.

10. Clarke, D.R., Materials selection guidelines for low thermal conductivity thermal barrier coatings. Surface and Coatings Technology. 2003, 163, 67-74.

11. Finkel, P., Seaman, B., Harrell, K.,Palma J., Hettinger, J., Lofland, S., Ganguly, A., Barsoum, M., Sun, Z. and Li, S., Electronic, thermal, and elastic properties of Ti 3 Si 1- x Ge x C 2 solid solutions. Physical Review B. 2004, 70(8), 085104.

12. Huang, B., McGaughey, A. and Kaviany, M., Thermal conductivity of metal-organic framework 5 (MOF-5): Part I. Molecular dynamics simulations. International journal of heat and mass transfer. 2007, 50(3), 393-404.

13. McGaughey, A. and Kaviany M., Thermal conductivity decomposition and analysis using molecular dynamics simulations: Part II. Complex silica structures. International Journal of Heat and Mass Transfer. 2004, 47(8), 1799-816.

14. Callaway, J., Quantum theory of the solid state: Elsevier, 1976, 57-75

15. Slack, G. A., Nonmetallic crystals with high thermal conductivity. Journal of Physics and Chemistry of Solids. 1973, 34(2), 321-35.

16. Ziman, J. M., Principles of the Theory of Solids: Cambridge university press, 1972, 220.

17. Aryal, S., Sakidja, R., Barsoum, M. W. and Ching, W. Y., A genomic approach to the stability, elastic, and electronic properties of the MAX phases. physica status solidi (b). 2014, 251(8), 1480-97.

18. Anderson, O. L., Using these elastic constants and J. Phys. Chem. Solids. 1963, 24, 909.

19. Schreiber, E., Anderson, O. L. and Soga, N., Elastic Constants and Their Measurement. McGrawHill, New York, 1973, 258-264. 
20. Luo, Y., Wang, J., Wang, J., Li, J. and Hu, Z., Theoretical Predictions on Elastic Stiffness and Intrinsic Thermal Conductivities of Yttrium Silicates. Journal of the American Ceramic Society. 2014, 97(3), 945-51.

21. Morelli, D. T. and Slack, G. A., High lattice thermal conductivity solids. High Thermal Conductivity Materials, Springer, 2006. p. 37-68.

22. Belomestnykh, V. and Tesleva, E., Interrelation between anharmonicity and lateral strain in quasi-isotropic polycrystalline solids. Technical physics. 2004, 49(8), 1098-100.

23. Sales, B., Chakoumakos, B., Mandrus, D., Sharp, J., Dilley, N. and Maple M., editors. Atomic Displacement Parameters, A Useful Tool in the Search for New Thermoelectric Materials, Materials Research Society Symposium Proceedings, 1999, MATERIALS RESEARCH SOCIETY.

24. Kittel, C., Wiley: Introduction to Solid State Physics., Wiley edition, 2004.

25. Julian, C. L., Theory of heat conduction in rare-gas crystals. Physical Review. 1965,137(1A), A128.

26. Clarke, D. R. and Phillpot, S. R., Thermal barrier coating materials. Materials Today. 2005, 8(6), 22-9.

27. Ziman, J., Electrons and Phonons: The Theory of Transport Phenomena in Solids, Clarendon. Oxford, 1960, 258-264.

28. Berman, R., Thermal Conductivity in Solids, Clarendon Press, Oxford, 1976.

29. Du, A., Wan, C., Qu, Z. and Pan, W., Thermal Conductivity of Monazite-Type REPO4 (RE= La, Ce, $\mathrm{Nd}, \mathrm{Sm}, \mathrm{Eu}, \mathrm{Gd}$ ). Journal of the American Ceramic Society. 2009, 92(11), 2687-92.

30. Abeles, B., Lattice thermal conductivity of disordered semiconductor alloys at high temperatures. Physical Review. 1963, 131(5), 1906.

31. Salama, I., El-Raghy, T. and Barsoum, M., Synthesis and mechanical properties of $\mathrm{Nb}<\mathrm{sub}>$ $2</$ sub $>$ AlC and $(\mathrm{Ti}, \mathrm{Nb})<\mathrm{sub}>2</$ sub $>$ AlC. Journal of alloys and compounds. 2002, 347(1), 2718.

32. Barsoum, M., El-Raghy, T., Rawn, C., Porter, W., Wang, H., Payzant, E. and Hubbard, C., Thermal properties of $\mathrm{Ti}<\mathrm{sub}>3</ \mathrm{sub}>\mathrm{SiC}<\mathrm{sub}>2</$ sub $>$. Journal of Physics and Chemistry of Solids. 1999, 60(4), 429-39. 


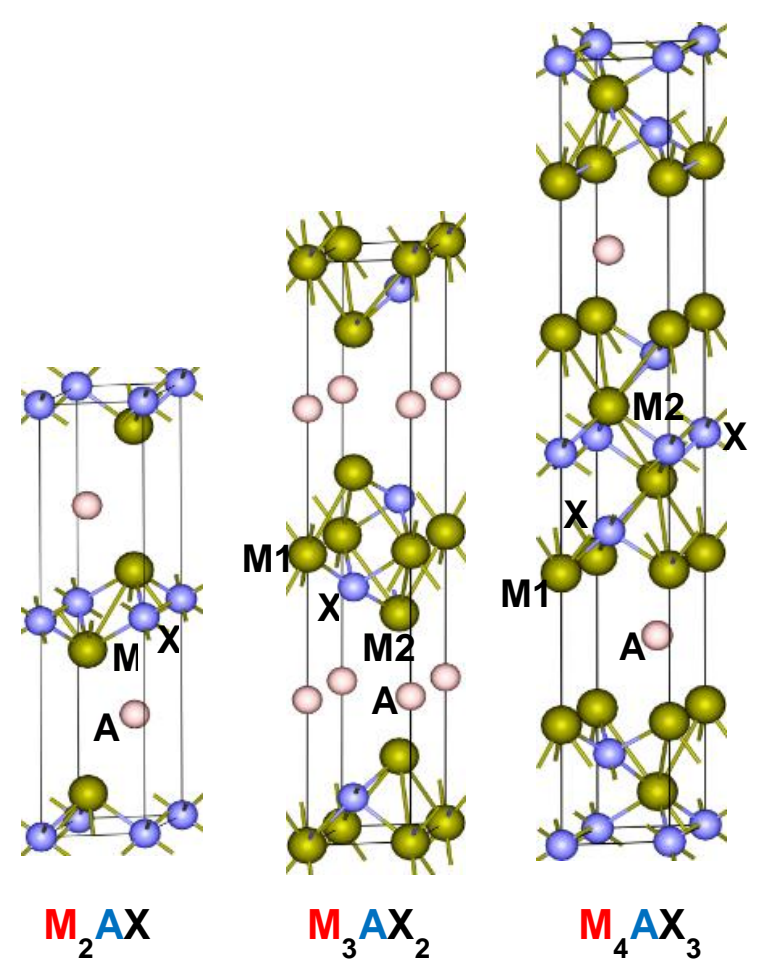

Figure 1

Figure

$M_{4} A X_{3}$ 

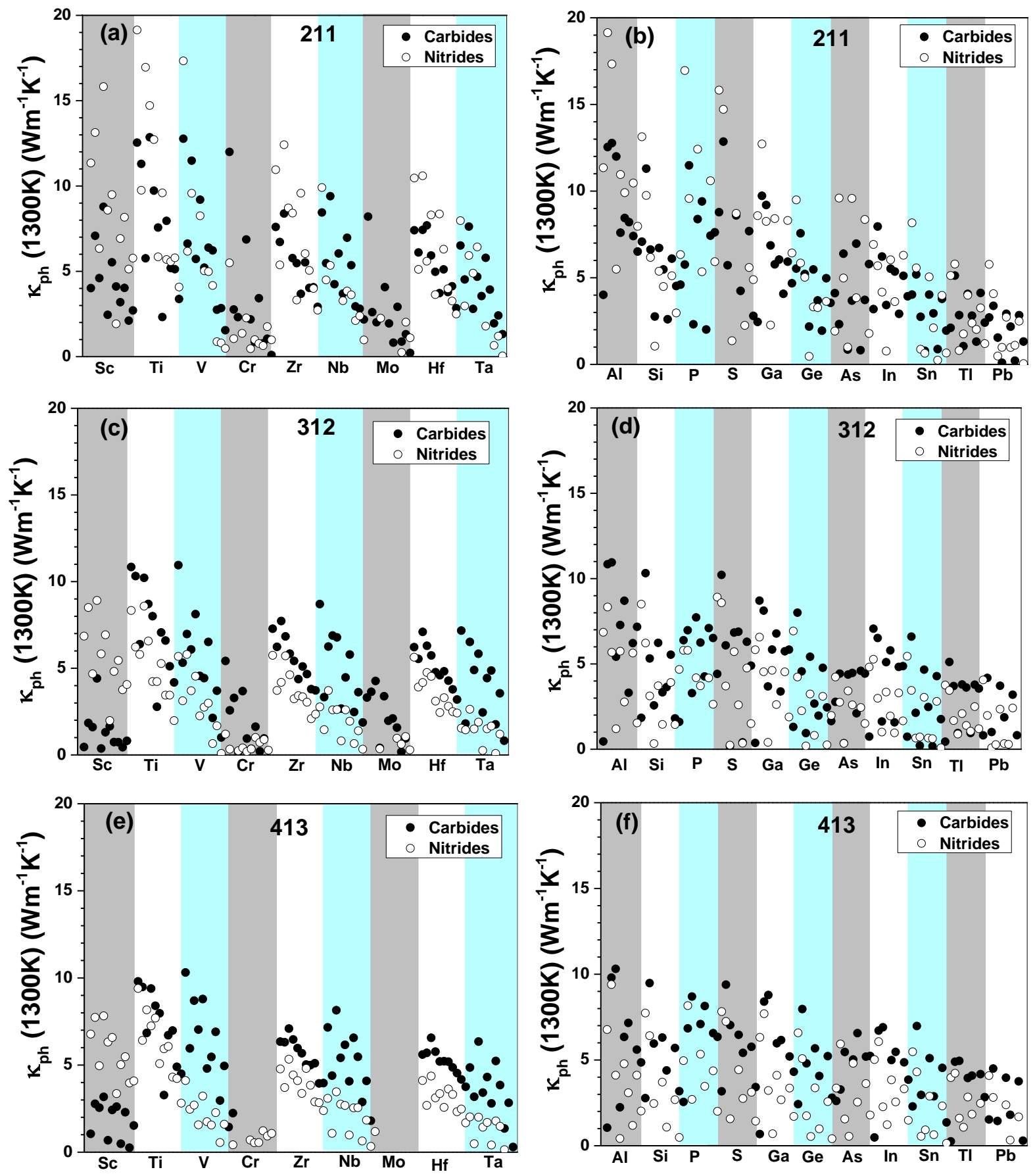

Figure 2 

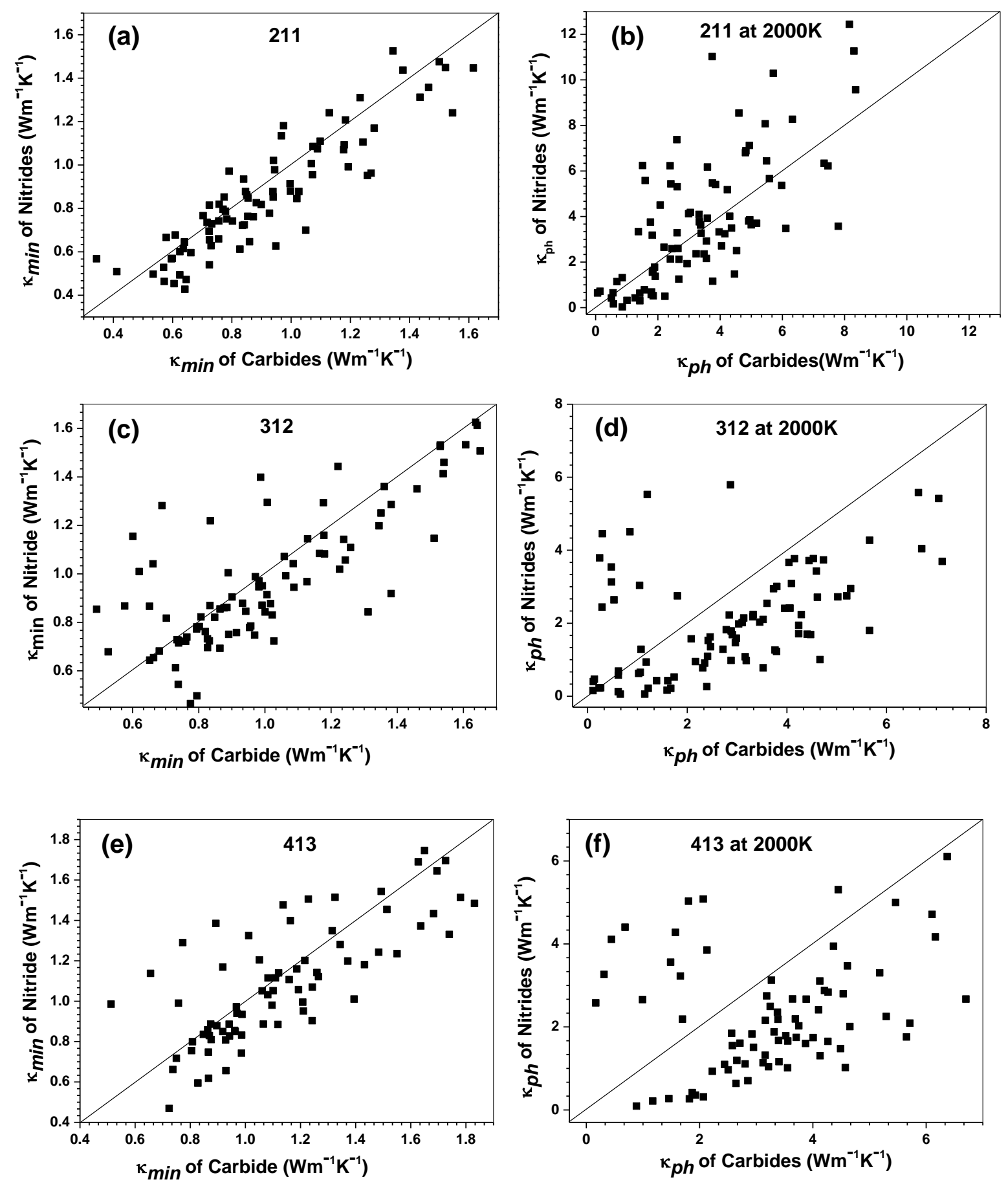

Figure 3 


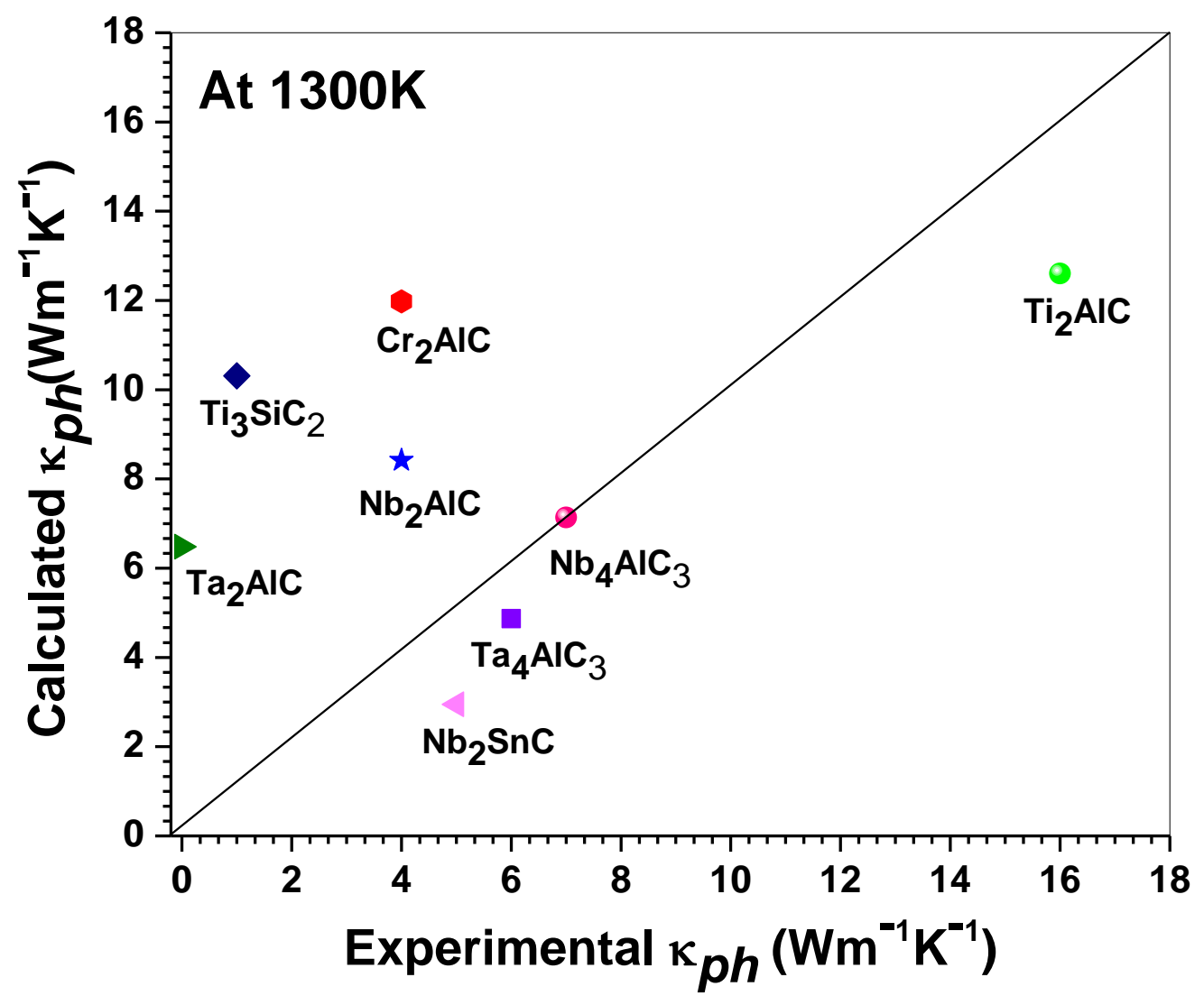

Figure 4 

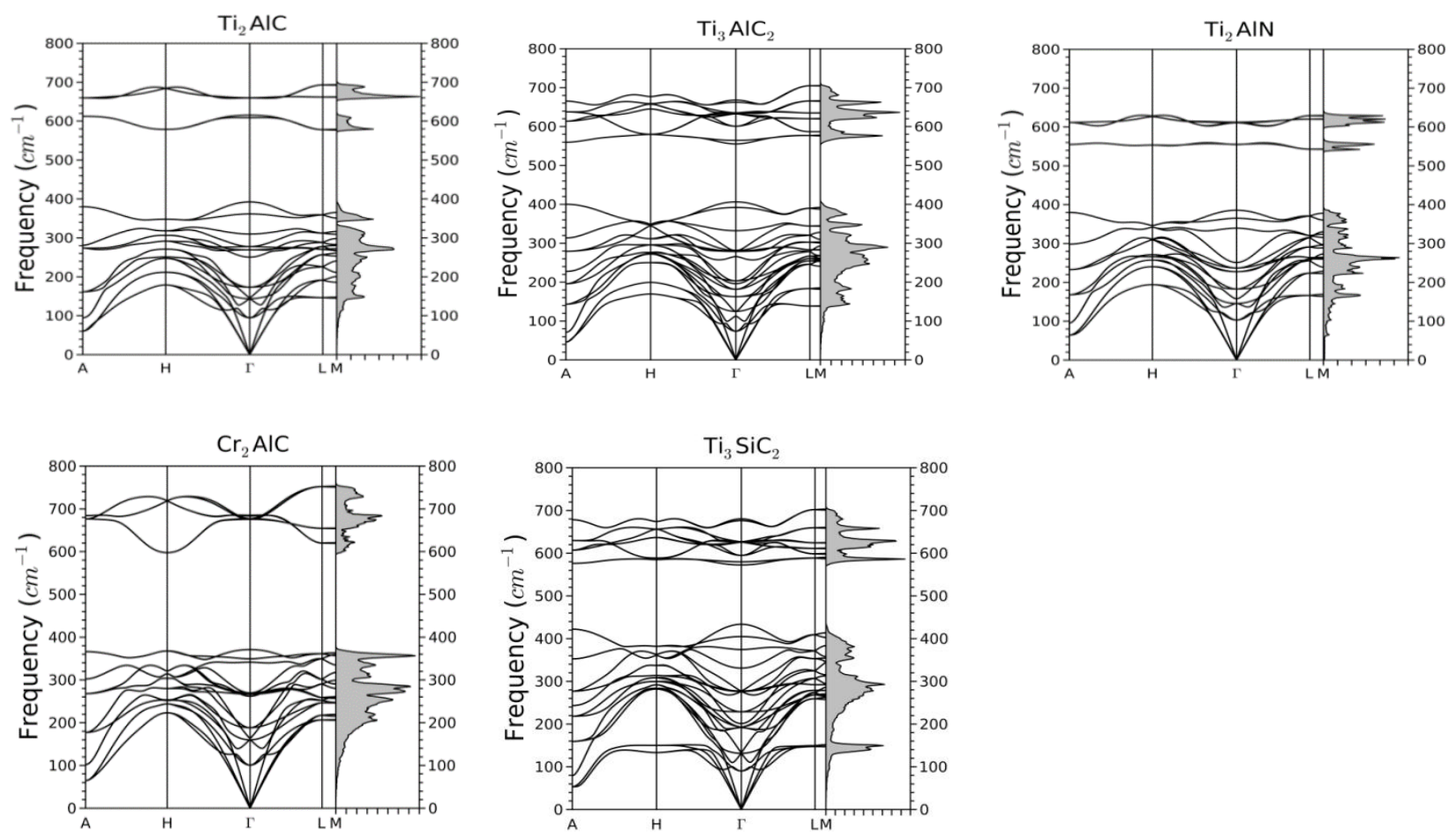

Figure 5 

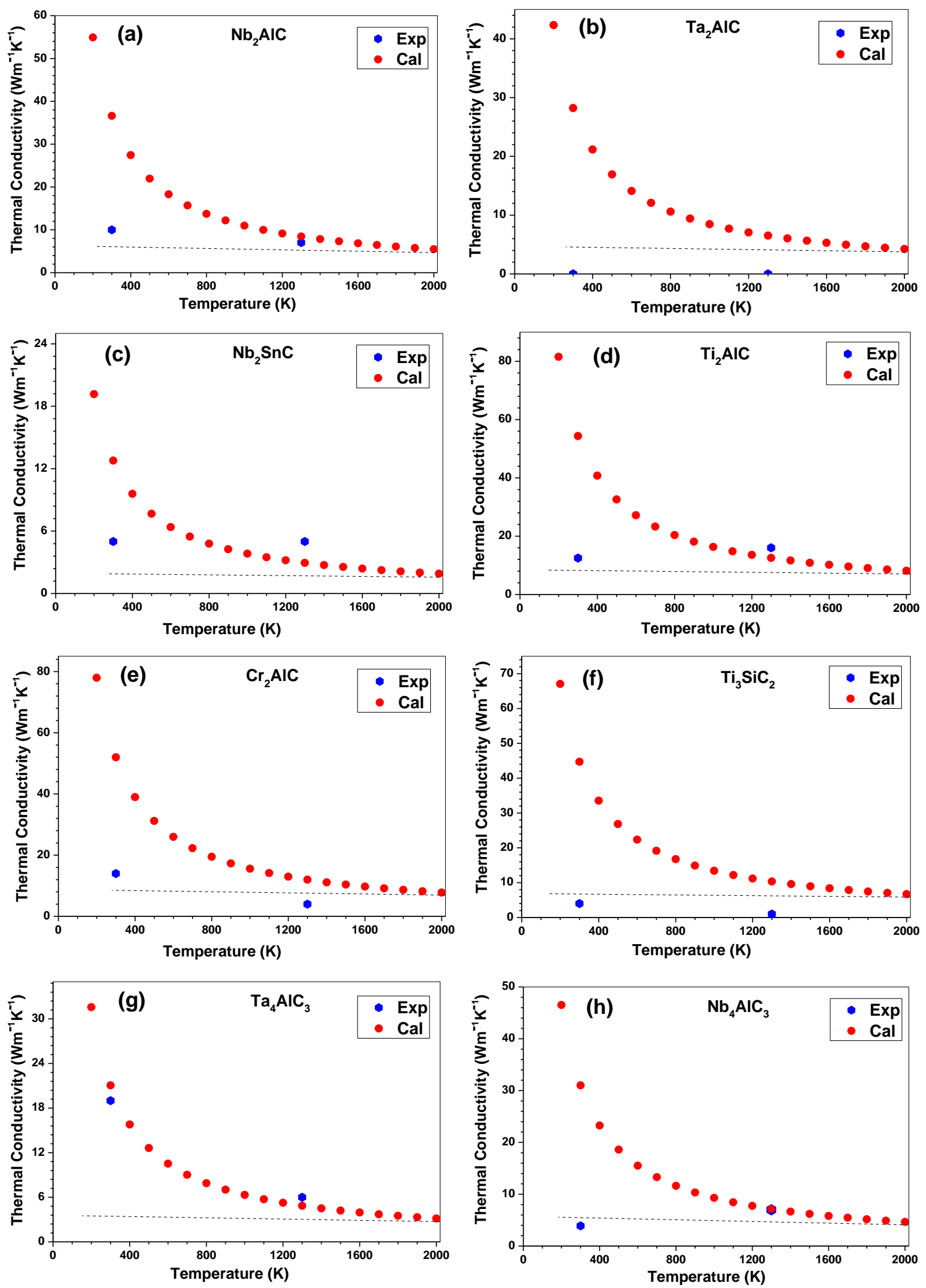

Figure 6 
Table 1. Calculated values of $\kappa_{\min }, \Theta_{\mathrm{D}}, \gamma, \kappa_{p h}$, volume, mass, number of atoms of unit cell of 8 MAX phases and experimental value of $\kappa_{p h}$.

\begin{tabular}{|c|l|l|l|l|l|c|c|l|}
\hline Compounds & $\mathrm{n}$ & $\Theta_{\mathrm{D}}(\mathrm{K})$ & $\Gamma$ & $\mathrm{Vol}\left(\AA^{3}\right)$ & $\mathrm{M}(\mathrm{amu})$ & $\begin{array}{c}\kappa_{p h}(\mathrm{cal}) \\
\left(\mathrm{Wm}^{-1} \mathrm{~K}^{-1}\right)\end{array}$ & $\begin{array}{l}\kappa_{p h}(\mathrm{exp}) \\
\left(\mathrm{Wm}^{-1} \mathrm{~K}^{-1}\right)\end{array}$ & $\begin{array}{l}\kappa_{\min } \\
\left(\mathrm{Wm}^{-1} \mathrm{~K}^{-1}\right)\end{array}$ \\
\hline $\mathrm{Ti}_{2} \mathrm{AlC}$ & 8 & 716 & 1.230 & 112.02 & 134.73 & 13 & 16 & 1.378 \\
\hline $\mathrm{Ta}_{2} \mathrm{AlC}$ & 8 & 449 & 1.448 & 115.58 & 400.89 & 7 & 0 & 0.855 \\
\hline $\mathrm{Nb}_{2} \mathrm{AlC}$ & 8 & 576 & 1.395 & 118.22 & 224.80 & 8 & $>4$ & 1.089 \\
\hline $\mathrm{Nb}_{2} \mathrm{SnC}$ & 8 & 412 & 1.689 & 129.35 & 316.53 & 3 & 5 & 0.755 \\
\hline $\mathrm{Cr}_{2} \mathrm{AlC}$ & 8 & 744 & 1.312 & 88.94 & 142.98 & 12 & 4 & 1.545 \\
\hline $\mathrm{Ti}_{3} \mathrm{SiC}_{2}$ & 12 & 795 & 1.324 & 145.25 & 195.70 & 10 & 1 & 1.607 \\
\hline $\mathrm{Ta}_{4} \mathrm{AlC}_{3}$ & 16 & 487 & 1.462 & 206.60 & 786.80 & 5 & 6 & 0.963 \\
\hline $\mathrm{Nb}_{4} \mathrm{AlC}_{3}$ & 16 & 644 & 1.374 & 210.71 & 434.64 & 7 & 7 & 1.265 \\
\hline
\end{tabular}

